Disponível em:

http://editora.unoesc.edu.br/index.php/race

RACE, Joaçaba, v. 16, n. 3, p. 885-910, set./dez. 2017

\title{
ANÁLISE FATORIAL DE SÉRIES TEMPORAIS PARA MEDIDAS DE LIQUIDEZ NO MERCADO BRASILEIRO
}

Times series factor analysis for liquidity measures on Brazilian stock market

Vinicius Girardi da Silveira

E-mail: vinicius.girardi@hotmail.com Graduado em Ciências Econômicas pela Universidade Federal de Santa Maria; Mestrando em Administração pela Universidade Federal de Santa Maria. Endereço para contato: Avenida Roraima, 1000, Cidade Universitária, Camobi, 97105-900, Santa Maria, Rio Grande do Sul, Brasil.

Kelmara Mendes Vieira

E-mail: kelmara@terra.com.br Doutora em Administração pela Universidade Federal do Rio Grande do Sul; Mestre em Administração pela Universidade Federal do Rio Grande do Sul; Professora Associada na Universidade Federal de Santa Maria.

Reisoli Bender Filho

E-mail: reisolibender@yahoo.com.br

Doutor em Economia Aplicada pela Universidade Federal de Viçosa; Mestre em Economia do Desenvolvimento pela Pontifícia Universidade Católica do Rio Grande do Sul; Professor Adjunto na Universidade Federal de Santa Maria.

Daniel Arruda Coronel

E-mail: daniel.coronel@uol.com.br

Doutor em Economia Aplicada pela Universidade Federal de Viçosa; Mestre em Agronegócios pela Universidade Federal do Rio Grande do Sul; Professor Adjunto na Universidade Federal de Santa Maria.

Artigo recebido em 13 de abril de 2017. Aceito em 12 de julho de 2017. 
Resumo

Neste estudo teve-se por objetivo sugerir o emprego de uma metodologia alternativa para mensuração da liquidez em mercados acionários. Para tanto, o procedimento empregado foi a Análise Fatorial de Séries Temporais (TSFA), proposta por Gilbert e Meijer (2005). Com base neste modelo, foram utilizados dados da negociação de ações da BM\&FBOVESPA de dezembro de 1994 a junho de 2011 para cinco diferentes proxies de liquidez que foram empregadas na construção fatorial. No estudo permitiu-se observar a possibilidade de se combinarem diferentes medidas de liquidez para a formação de um único fator que demonstrou possuir uma forte associação com as proxies utilizadas em sua construção. Como vantagens, esse procedimento permite eliminar problemas de colinearidade em estimações estatísticas e possibilita que análises de investimento utilizem uma única variável para mensurar a liquidez ao invés de várias simultaneamente. Além disso, a construção fatorial pode ser replicada para novos dados, já que não é assumido um modelo dinâmico para os fatores, o qual é tratado simplesmente como um problema de mensuração.

Palavras-chave: Liquidez. Análise Fatorial de Séries Temporais. Mercado acionário brasileiro.

Abstract

The purpose of this paper is to suggest a new methodology to measure liquidity in stock markets. For that, we use the Times Series Factor Analysis model (TSFA), proposed by Gilbert and Meijer (2005). We employed on that model, data from December, 1994 to June, 2011 of five liquidity proxies. As findings, we observe that is possible to combine liquidity measures to create a unique factor, which show to have a high correlation with the liquidity proxies. The advantages of this procedure is to eliminate the problem of collinearity on statistical estimations and the possibility of use a single variable to measure liquidity in investment analysis. Furthermore, the factorial construction can be replicated for new datas, since is not assumed a dynamic model for the factors, which is treated simply as a measurement procedure.

Keywords: Liquidity. Times Series Factor Analysis. Brazilian stock market.

\section{INTRODUÇÃO}

O processo de globalização possibilitou um intenso intercâmbio cultural e econômico entre os países, fazendo com que o mercado acionário adquirisse um importante papel no cenário financeiro internacional. Tendo em vista as oportunidades acompanhadas por esse processo, países, tanto desenvolvidos como emergentes, procuraram abrir suas economias a fim de receber novos investimentos. Essa mobilidade de capitais tornou o mercado acionário uma importante fonte de captação de recursos, permitindo o desenvolvimento de empresas e contribuindo para o progresso dos países. 
Tal crescimento do mercado acionário vem fomentando pesquisadores a buscar respostas acerca dos fatores que influenciam os diferentes aspectos da negociação dos ativos financeiros. Desse modo, um amplo desafio tanto aos pesquisadores, quanto aos profissionais e investidores que se utilizam desse mercado é identificar e compreender os aspectos que influenciam o seu comportamento.

Entre esses fatores se destaca a liquidez, sendo um atributo crucial quando da análise de ativos de capital, influenciando sua precificação. Para Amihud e Mendelson (1991), o efeito liquidez pode ser comparado ao efeito risco sobre ativos de capital. Investidores avessos ao risco exigem maiores retornos esperados como compensação pelo risco maior. De forma similar, investidores preferem investir em ativos líquidos que possam ser transacionados rapidamente e com baixo custo.

Um ativo é considerado líquido se puder ser negociado rapidamente, em grandes quantidades, a um baixo custo e com pouco impacto em seu preço (LIU, 2006). Ativos pouco líquidos tendem a exigir um custo de transação maior quando da sua compra e venda e, para que possam ser atrativos quando comparados a ativos mais líquidos, devem oferecer um retorno maior para que os investidores se sintam atraídos em mantê-los em suas carteiras.

Tem-se que a liquidez dos ativos afeta de forma intensa o mercado acionário, influenciando na precificação dos ativos e afetando o risco dos investimentos. No entanto, conforme enfatizam Pastor e Stambaugh (2003), ainda não há um conceito de liquidez teoricamente correto ou universalmente aceito, nem uma medida que consiga captar todas as suas múltiplas dimensões.

Assim, os estudos sobre o tema têm empregado diversas medidas que buscam mensurar a liquidez dos ativos, com estas captando os diferentes aspectos das negociações. Nesse sentido, este artigo tem por objetivo sugerir o emprego de uma metodologia alternativa para mensuração da liquidez em mercados acionários. Para tanto, o procedimento empregado foi a Análise Fatorial de Séries Temporais (TSFA), proposta por Gilbert e Meijer (2005), a qual serve como uma alternativa capaz de criar um fator que abranja as proxies existentes na literatura.

A escolha desse objetivo de pesquisa se deve, em suma, à liquidez acionária possuir diferentes dimensões, ou seja, a liquidez ou iliquidez de uma ação ou de um mercado costuma ser representada de diferentes formas. Alguns autores como Amihud (2002) optam por considerar o impacto no preço ao negociar um ativo como representativo da iliquidez. Outros, como Liu (2006), adotam em seus estudos medidas referentes à velocidade de negociação, ou seja, à continuidade de um negócio e ao potencial atraso ou dificuldade em executar uma ordem. Já autores como Chordia, 
Roll e Subrahmanyam (2000) consideram a liquidez o volume financeiro ou o número de negócios realizados com uma ação.

Visto que qualquer investidor, na compra ou na venda de ações, está exposto ao risco de liquidez, esta deve ser considerada quando da tomada de decisões de investimento. No entanto, sua multiplicidade conceitual acaba por confundir investidores e pesquisadores, que divergem sobre a melhor forma de mensurar a liquidez ou mesmo empregam simultaneamente diferentes formas. Assim, este artigo tem como enfoque analisar a liquidez a partir de uma metodologia que permita que essa característica do mercado financeiro possa ser representada por uma única variável ao invés de várias simultaneamente.

A seguir, apresenta-se um breve embasamento teórico abordando algumas definições sobre liquidez, além de estudos realizados sobre o tema. Na sequência, são abordados os procedimentos metodológicos da pesquisa e os resultados obtidos. Por fim, apresentam-se as considerações finais.

\section{REVISÃO BIBLIOGRÁFICA}

Esta seção tem o objetivo de expor estudos feitos sobre liquidez acionária, destacando as respectivas proxies de liquidez utilizadas pelos autores. Liquidez, em termos econômicos, é definida como a propriedade do que é facilmente negociável e convertível em dinheiro vivo (HOUAISS; VILLAR, 2009). Com base na multiplicidade do conceito de liquidez, Liu (2006) desenvolve um conceito que engloba múltiplas dimensões. $\mathrm{O}$ autor considera que um ativo é líquido se puder ser negociado rapidamente em grandes quantidades, a um baixo custo e com pouco impacto em seu preço.

Além da definição de um ativo líquido, existem as definições de um mercado líquido. Black (1971) descreve um mercado líquido como aquele que apresenta as seguintes condições: sempre há preços de compra e venda para o investidor que deseja comprar ou vender uma pequena quantidade de ações imediatamente; a diferença entre os preços de compra e venda (spread) é sempre pequena; um investidor que deseja comprar ou vender uma grande quantidade de ações, na ausência de informações especiais, poderá esperar para negociar por um longo período de tempo por um preço não muito diferente, em média, do preço de mercado corrente; e um investidor pode comprar ou vender uma grande quantidade de ações imediatamente, mas com um prêmio ou desconto que depende do tamanho do negócio.

Em outras palavras, um mercado líquido é um mercado contínuo e eficiente. Contínuo no sentido de que quase qualquer quantidade de ações pode ser comprada ou 
vendida imediatamente. Eficiente, no sentido de que pequenas quantidades de ações podem ser sempre compradas e vendidas a um valor muito próximo do preço de mercado corrente.

Kyle (1985) afirma que o conceito de um mercado líquido é escorregadio e ilusório, já que engloba diversas propriedades transacionais dos mercados, como tightness (custo de mudar uma posição em um curto período de tempo), depth (tamanho de uma ordem capaz de mudar o preço das ações) e resiliency (velocidade com que os preços se recuperam após um choque inesperado). Sarr e Lybek (2002) complementam essa definição englobando immediacy (velocidade com que uma ordem pode ser executada) e breadth (movimentação de uma grande quantidade de negócios ou um grande volume a um mínimo impacto no preço) como características de um mercado líquido.

Dada essa abrangência multidimensional no que diz respeito à liquidez, a literatura especializada vem empregando diferentes medidas. Algumas estão relacionadas à atividade de negociação e se referem à quantidade de negócios, de ações e ao volume financeiro negociado. O turnover, razão entre a quantidade de ações negociadas e a quantidade de ações emitidas, é uma medida relacionada à atividade de negociação. O bid-ask spread, diferença entre o preço máximo que um comprador está disposto a pagar por um determinado ativo e o preço mínimo que um investidor está disposto a vender, diz respeito ao custo de negociação. A medida Iliquidez de Amihud (2002) se caracteriza por apresentar a dimensão de impacto no preço, o que expressa a sensibilidade do retorno de um ativo ao volume financeiro negociado em determinado período.

Amihud e Mendelson (1986) afirmam que a importância da liquidez está relacionada diretamente ao custo do capital, que pode ser diminuído por meio de políticas financeiras que aumentem a liquidez. O estudo desses autores foi um dos primeiros a analisar o papel da liquidez na precificação dos ativos no mercado americano, sugerindo uma relação positiva entre retorno e iliquidez. Eles utilizaram como proxy de liquidez o bid-ask spread e concluíram que o excesso de retorno esperado pelo mercado é função crescente e côncava do spread das ações.

Datar, Naik e Radcliffe (1998) também averiguaram a relação entre retorno e liquidez para o mercado acionário americano. Os autores estudaram o período de 1962 a 1991, a partir da medida turnover (razão entre a quantidade de ações negociadas e a quantidade de ações em circulação) como uma alternativa à utilização do bid-ask spread, como sugerido por Amihud e Mendelson (1986). Como resultado, 
constataram que a liquidez medida pelo turnover possuía um importante papel na explicação das variações nos retornos acionários.

A relação entre iliquidez e retorno acionário das empresas também foi objeto de estudo de Amihud em 2002. O autor analisou essa relação no mercado americano para o período de 1964 a 1997 e utilizou uma medida denominada Iliquidez, dada pela razão entre o retorno absoluto diário e o volume em dólares, investigando a relação tanto para cross-section quanto para série temporal. Como resultado, o autor demonstrou que a iliquidez tem um efeito positivo e altamente significativo sobre os retornos esperados.

Pastor e Stambaugh (2003) analisaram o risco de liquidez para o mercado americano em um período de 34 anos. Como resultado, observaram que ativos que possuíam maior risco de liquidez também registravam retornos superiores aos demais ativos, sendo esse retorno adicional definido como prêmio de liquidez. Ademais, constataram que as maiores depressões na liquidez ocorreram em meses perfeitamente identificáveis com importantes eventos econômicos e financeiros, como o embargo do petróleo em 1973 e as crises financeiras no final da década de 1990.

Usando uma medida de liquidez, que capta sua natureza multidimensional, Liu (2006) desenvolveu um modelo que incorpora a liquidez ao tradicional modelo de precificação de ativos de capital, o CAPM. O autor utiliza como proxy para liquidez o turnover padronizado e ajustado para o número de dias sem volume de negociação. Ele observou que seu modelo capta um prêmio de liquidez que o CAPM e o modelo de três fatores de Fama e French (1993) falham em evidenciar. Adicionalmente, constata que os maiores declínios na liquidez no mercado acionário americano ocorreram durante grandes choques econômicos ou financeiros.

Chordia, Roll e Subrahmanyam (2000), em um estudo pioneiro sobre comunalidades, sugerem que a existência de movimentos correlacionados durante as negociações de ativos levaria à comunalidade na liquidez. Os autores analisaram a existência de co-movimentos na liquidez de ações individuais negociadas na New York Stock Exchange (NYSE), por meio de dados diários para o ano 1992. Empregaram o quoted-spreads, quoted-depth e spreads efetivos como medidas de liquidez, além da volatilidade, volume e preço das ações como variáveis de controle. As principais constatações dos autores foram de que a liquidez individual das ações se co-movimenta com a liquidez geral do mercado e do setor no qual uma determinada empresa atua.

Quanto às investigações sobre liquidez acionária no Brasil, Bruni e Famá (1998) utilizaram dados da Bovespa do período de 1988 a 1997 e um modelo de dois fatores, incorporando a liquidez, medida pela negociabilidade da ação, ao CAPM. Os 
autores formaram 25 portfólios de acordo com o risco sistemático e liquidez de cada ativo individual, empregando as carteiras em regressões cross-section. Os resultados demonstraram uma associação negativa e significativa entre liquidez e retorno acionário, sugerindo a existência de um prêmio por liquidez no mercado brasileiro. Adicionalmente, constataram que a liquidez apresentou níveis de significância maiores do que o próprio risco sistemático, o que demonstraria a importância da análise da liquidez sobre o risco sistemático no mercado acionário brasileiro.

Minardi, Sanvicente e Monteiro (2005) também investigaram o mercado brasileiro no período compreendido entre 1998 e 2003. Os autores analisaram a relação do bid-ask spread relativo (ou simplesmente spread, sendo o bid-ask spread em reais dividido pela média entre a última melhor cotação de compra e a última melhor cotação de venda), com o retorno das ações e com a assimetria de informações. Foram realizadas regressões aparentemente não relacionadas (SUR - seemingly unrelated regressions) aos dados em painel. Quanto aos ativos individuais, os resultados mostraram uma relação negativa e significante entre o retorno das ações e iliquidez, sugerindo um prêmio de liquidez negativo. Já em relação às carteiras de ativos, na tentativa de validar o resultado anterior, a relação apresentou-se negativa, porém, não significativa. A existência de prêmio por liquidez negativo no Brasil, apesar de não validado, mostrou-se um resultado contrário ao encontrado no mercado americano e ao que seria esperado. Os autores, ainda, observaram que o custo de transações é menor à medida que melhora a transparência das informações.

Dando continuidade aos estudos no Brasil, Vieira e Milach (2008) estudaram o comportamento da liquidez/iliquidez no mercado brasileiro, no período de janeiro de 1995 a junho de 2005, utilizando regressões múltiplas, com base na metodologia de Fama e Macbeth (1973). Os autores utilizaram seis medidas de liquidez (volume, quantidade de títulos, quantidade de negócios, turnover, spread e iliquidez) e três variáveis de controle (valor de mercado, volatilidade e dividend yield - rentabilidade dos dividendos). A maioria dos coeficientes das proxies empregadas não foi significativa, apenas as variáveis relacionadas à iliquidez, como Iliquidez de Amihud (2002) e spread, foram significativas. Assim, os testes efetuados sugeriram a existência de uma relação entre retorno e iliquidez no mercado brasileiro para o período estudado, evidenciando um prêmio por liquidez.

O papel da liquidez na formação dos preços de ativos financeiros brasileiros também foi verificado por Correia, Amaral e Bressan (2008), no período de 1995 a 2004, utilizando o retorno das ações negociadas na Bovespa. As medidas de liquidez utilizadas foram o volume e a quantidade de negócios e o turnover, empregando-se a 
técnica de regressão múltipla de dados em painel por meio dos modelos de equações aparentemente não relacionadas (SUR). Os resultados indicaram uma relação linear e positiva entre retorno e variáveis relacionadas à atividade de negociação, as quais são o volume, a quantidade de negócios e o turnover, não sendo observado um prêmio de liquidez no mercado acionário brasileiro.

Machado e Medeiros (2011) averiguaram se a liquidez é precificada e se explica parcialmente as variações dos retornos das ações no mercado brasileiro no período de junho de 1995 a junho de 2008. Os autores utilizaram como medidas de liquidez o turnover, o volume, a quantidade de negócios, a negociabilidade e o turnover padronizado e optaram pelo emprego de portfólios. Fazendo uso do CAPM, juntamente com o modelo de três (CAPM mais tamanho da empresa medido pelo valor de mercado e índice book-to-market, definido pela relação entre valor contábil e de mercado de determinada empresa), quatro (modelo de três fatores mais o fator momento) e cinco fatores (modelo de quatro fatores mais o fator liquidez), constataram que tanto a hipótese de que existe prêmio por liquidez quanto a de que a liquidez é precificada e explica parte das variações dos retornos no mercado brasileiro não foram rejeitadas.

Righi e Vieira (2012) investigaram a relação entre retorno e liquidez no mercado acionário brasileiro para o período de janeiro de 2002 a abril de 2010, considerando diferentes escalas de tempo por meio da decomposição em ondaletas (wavelets). Os autores utilizaram como proxy de liquidez a medida diária de Amihud (2002), calculada pelo índice Bovespa, e fizeram uso de regressão múltipla. Os resultados obtidos indicaram um relacionamento positivo e significativo entre retorno e iliquidez, indicando, assim, que existiria um prêmio de liquidez para investidores que manterem ativos ilíquidos em suas carteiras.

Correia e Amaral (2012) analisaram o efeito da liquidez sobre a rentabilidade das ações negociadas na Bolsa de Valores de São Paulo entre 1995 e 2010, fazendo uso de regressões de dados em painel para a análise das ações divididas em diferentes carteiras. As medidas de liquidez utilizadas foram bid-ask spread, quoted spread, spread efetivo, volume de negociação e turnover, além de um índice de liquidez. Como resultados constataram um prêmio por liquidez apenas no período em que a crise financeira de 2008 se mostrou mais intensa. Além disso, constataram que as medidas de volume financeiro e turnover apresentaram uma associação positiva com a taxa de retorno ajustada ao risco, indicando que a liquidez pode funcionar como um fator de risco.

Em estudo posterior, Machado e Medeiros (2012) analisaram se existe o efeito liquidez no mercado acionário brasileiro e avaliaram a capacidade do CAPM e do 
modelo de três fatores de Fama e French (1993) em explicá-lo, por meio de dados de ações negociadas na Bovespa no período compreendido entre 1995 e 2008. Novamente, utilizaram como medidas de liquidez: turnover, volume, quantidade de negócios, negociabilidade e turnover padronizado, e optaram pelo emprego de portfólios. Os resultados permitiram concluir que existe um prêmio de liquidez no mercado brasileiro, independente da proxy utilizada, não havendo grandes alterações ao serem utilizados períodos distintos na análise. Adicionalmente, também constataram uma associação positiva e significativa entre o retorno e medidas consideradas de liquidez, o que é contrário ao esperado.

Victor, Perlin e Mastella (2013) estudaram a presença de comunalidades na liquidez de 30 ações negociadas na bolsa de valores brasileira no período de 2010 a 2012. Calcularam a média dos valores referentes à atividade de negociação, construindo variáveis que representam o valor médio da negociação de ativos para um período de 15 minutos e obtendo, assim, um conjunto de dados intraday. Como metodologia, utilizaram o volume negociado e o número de negócios como proxy de liquidez e estimaram regressões individuais para cada um dos ativos, tendo como base o modelo proposto por Chordia, Roll e Subrahmanyam (2000). Como resultado, constataram evidências de que as mudanças de liquidez dos ativos individuais são relacionadas às mudanças de liquidez do mercado como um todo.

Machado e Machado (2014) analisaram se o modelo de dois fatores desenvolvido por Liu (2006) explica as variações dos retornos das ações no mercado acionário brasileiro, tendo como objetivo secundário comparar o desempenho do modelo de dois fatores de Liu com o do CAPM e com o de três fatores de Fama e French (1993), bem como investigar se o modelo é robusto às estratégias baseadas nos efeitos tamanho da empresa, book-to-market, estratégia do momento, lucro/preço, fluxo de caixa/preço, liquidez e alavancagem, denominadas anomalias de valor. Para o desenvolvimento do estudo, os autores optaram pelo emprego de portfólios, e, para analisar o desempenho do modelo na explicação das variações dos retornos das ações, foram utilizadas regressões múltiplas. A população consistiu de todas as empresas com ações listadas na BM\&FBOVESPA, no período de 1995 a 2008. Como resultados principais, foi percebida uma melhora no poder explicativo do modelo de dois fatores em relação ao CAPM e um desempenho muito próximo ao modelo de três fatores.

Vieira, Justen Júnior e Righi (2015) analisaram a influência da liquidez no retorno das ações no mercado acionário brasileiro, empregando a medida proposta por Amihud (2002), além das medidas tradicionais, como o volume financeiro, a quantidade de negócios, o spread e o turnover, e utilizaram dados de dezembro de 1994 a 
abril de 2010 de ações negociadas na BM\&FBOVESPA. Os resultados obtidos possibilitaram concluir que a iliquidez de mercado esperada, assim como a variável spread apresentam um impacto positivo e estatisticamente significativo no retorno das ações. Já a iliquidez inesperada de mercado, bem como a variável quantidade de negócios têm um impacto negativo e estatisticamente significativo no retorno.

No que segue, é apresentado um resumo dos estudos discutidos (Quadro 1).

Quadro 1 - Resumo dos trabalhos pesquisados e suas respectivas proxies de liquidez

\begin{tabular}{|c|c|c|}
\hline Autores & Proxies & O que estudou? \\
\hline $\begin{array}{l}\text { Amihud e Mendel- } \\
\text { son (1986) }\end{array}$ & Bid-ask spread & $\begin{array}{l}\text { O papel da liquidez na precificação dos } \\
\text { ativos no mercado americano. }\end{array}$ \\
\hline $\begin{array}{l}\text { Datar, Naik e Rad- } \\
\text { cliffe (1998) }\end{array}$ & Turnover & $\begin{array}{l}\text { A relação entre retorno e liquidez para o } \\
\text { mercado acionário americano. }\end{array}$ \\
\hline Amihud (2002) & Iliquidez de Amihud (2002) & $\begin{array}{l}\text { A relação entre iliquidez e retorno acionário } \\
\text { das empresas no mercado americano. }\end{array}$ \\
\hline $\begin{array}{l}\text { Pastor e Stambaugh } \\
\text { (2003) }\end{array}$ & $\begin{array}{l}\text { Gamma de Pastor e Stambaugh } \\
\text { (2003) }\end{array}$ & $\begin{array}{l}\text { O risco de liquidez para o mercado ameri- } \\
\text { cano. }\end{array}$ \\
\hline Liu (2006) & Turnover padronizado & $\begin{array}{l}\text { Desenvolveu um modelo que incorpora a } \\
\text { liquidez ao CAPM. }\end{array}$ \\
\hline $\begin{array}{l}\text { Chordia, Roll e } \\
\text { Subrahmanyam } \\
\text { (2000) }\end{array}$ & $\begin{array}{l}\text { Quoted-spreads, quoted-depth e } \\
\text { spreads efetivos e volume }\end{array}$ & $\begin{array}{l}\text { A existência de movimentos correlacionados } \\
\text { durante as negociações de ativos que leva- } \\
\text { riam à comunalidade na liquidez. }\end{array}$ \\
\hline $\begin{array}{l}\text { Bruni e Famá } \\
\text { (1998) }\end{array}$ & $\begin{array}{l}\text { Índice de negociabilidade (Bo- } \\
\text { vespa) }\end{array}$ & $\begin{array}{l}\text { Um modelo de dois fatores, incorporando a } \\
\text { liquidez ao CAPM }\end{array}$ \\
\hline $\begin{array}{l}\text { Minardi, Sanvicente } \\
\text { e Monteiro (2005) }\end{array}$ & Spread & $\begin{array}{l}\text { A relação do bid-ask spread relativo, com } \\
\text { o retorno das ações e com a assimetria de } \\
\text { informações. }\end{array}$ \\
\hline $\begin{array}{l}\text { Vieira e Milach } \\
\text { (2008) }\end{array}$ & $\begin{array}{l}\text { Volume, quantidade de títulos, } \\
\text { quantidade de negócios, turno- } \\
\text { ver, spread e Iliquidez }\end{array}$ & $\begin{array}{l}\text { O comportamento da liquidez/iliquidez no } \\
\text { mercado brasileiro por meio da metodologia } \\
\text { de Fama e Macbeth (1973). }\end{array}$ \\
\hline $\begin{array}{l}\text { Correia, Amaral e } \\
\text { Bressan (2008) }\end{array}$ & $\begin{array}{l}\text { Turnover, quantidade de negó- } \\
\text { cios e volume }\end{array}$ & $\begin{array}{l}\text { O papel da liquidez na formação dos preços } \\
\text { de ativos financeiros brasileiros. }\end{array}$ \\
\hline $\begin{array}{l}\text { Machado e Medei- } \\
\text { ros (2011) }\end{array}$ & $\begin{array}{l}\text { Turnover, volume, quantidade } \\
\text { de negócios, negociabilidade,e } \\
\text { turnover padronizado }\end{array}$ & $\begin{array}{l}\text { Averiguaram se a liquidez é precificada e se } \\
\text { explica parcialmente as variações dos retor- } \\
\text { nos das ações no mercado brasileiro. }\end{array}$ \\
\hline $\begin{array}{l}\text { Righi e Vieira } \\
\text { (2012) }\end{array}$ & Iliquidez de Amihud (2002) & $\begin{array}{l}\text { A relação entre retorno e liquidez no mer- } \\
\text { cado acionário através da decomposição em } \\
\text { ondaletas (wavelets). }\end{array}$ \\
\hline $\begin{array}{l}\text { Correia e Amaral } \\
\text { (2012) }\end{array}$ & $\begin{array}{l}\text { Bid-ask spread, quoted spread, } \\
\text { spread efetivo, volume de ne- } \\
\text { gociação, turnover, índice de } \\
\text { liquidez }\end{array}$ & $\begin{array}{l}\text { O efeito da liquidez sobre a rentabilidade } \\
\text { das ações negociadas na Bolsa de Valores de } \\
\text { São Paulo. }\end{array}$ \\
\hline $\begin{array}{l}\text { Machado e Medei- } \\
\text { ros (2012) }\end{array}$ & $\begin{array}{l}\text { Turnover, volume, quantidade } \\
\text { de negócios, negociabilidade e } \\
\text { turnover padronizado }\end{array}$ & $\begin{array}{l}\text { Se existe o efeito liquidez no mercado acio- } \\
\text { nário brasileiro e avaliaram a capacidade do } \\
\text { CAPM e do modelo de três fatores de Fama } \\
\text { e French (1993) em explicá-lo. }\end{array}$ \\
\hline
\end{tabular}




\begin{tabular}{|l|l|l|}
\hline \multicolumn{1}{|c|}{ Autores } & \multicolumn{1}{|c|}{ Proxies } & \multicolumn{1}{c|}{ O que estudou? } \\
\hline $\begin{array}{l}\text { Victor, Perlin e } \\
\text { Mastella (2013) }\end{array}$ & $\begin{array}{l}\text { Volume negociado e número de } \\
\text { negócios }\end{array}$ & $\begin{array}{l}\text { A presença de comunalidades na liquidez } \\
\text { de 30 ações negociadas na bolsa de valores } \\
\text { brasileira. }\end{array}$ \\
\hline $\begin{array}{l}\text { Machado e Macha- } \\
\text { do (2014) }\end{array}$ & $\begin{array}{l}\text { Turnover padronizado de Liu } \\
\text { (2006) }\end{array}$ & $\begin{array}{l}\text { Se o modelo de dois fatores desenvolvido } \\
\text { por Liu (2006) explica as variações dos } \\
\text { retornos das ações no mercado acionário } \\
\text { brasileiro. }\end{array}$ \\
\hline $\begin{array}{l}\text { Vieira, Justen e } \\
\text { Righi (2015) }\end{array}$ & $\begin{array}{l}\text { Iliquidez de Amihud (2002), } \\
\text { volume financeiro, quantidade } \\
\text { de negócios, spread e turnover }\end{array}$ & $\begin{array}{l}\text { A influência da liquidez no retorno das ações } \\
\text { no mercado acionário brasileiro. }\end{array}$ \\
\hline Fonte: os autores. & \multicolumn{2}{|l}{} \\
\hline
\end{tabular}

Ao se observar o Quadro 1, independentemente do objetivo, percebe-se que a decisão sobre qual proxy de liquidez utilizar ainda se mostra um desafio nas pesquisas sobre liquidez. É nesse ponto que este trabalho busca propor uma alternativa de mensuração, por meio de uma construção fatorial generalizável e capaz de representar a liquidez acionária.

\section{MÉTODO}

\subsection{DELINEAMENTO}

Esta seção apresenta os procedimentos metodológicos empregados para a construção de um fator de liquidez. Assim, para a formação da amostra foram consideradas as empresas registradas como sociedades anônimas de capital aberto, com ações negociadas na Bolsa de Valores, Mercadorias e Futuros de São Paulo (BM\&FBOVESPA) no período compreendido entre dezembro de 1994 e junho de 2011. A escolha por esse período deve-se à estabilidade inflacionária obtida pós-Plano Real, que se manteve até o momento em que os dados foram coletados em junho de 2011. Para o período compreendido na amostra foram empregados dados mensais das ações negociadas na BM\&FBOVESPA, visando à formação das medidas utilizadas nos modelos testados. Não fazem parte da análise aquelas ações que não apresentaram nenhuma negociação durante o período analisado. Assim, a amostra é composta por um painel de dados que perfaz 485 ações em 199 períodos, visto que nem todas as ações negociaram em todos os períodos estudados.

Optou-se por empregar na construção fatorial cinco proxies de liquidez comumente utilizadas pela literatura e que não exigem muito esforço para sua construção, as quais estão apresentadas no Quadro 2. 
Quadro 2 - Definição das variáveis utilizadas para a construção fatorial

\begin{tabular}{|l|l|}
\hline \multicolumn{1}{|c|}{ Variável } & \multicolumn{1}{c|}{ Definição } \\
\hline Bid-Ask Spread & $\begin{array}{l}\text { Diferença entre o preço máximo e o preço mínimo em que uma ação foi } \\
\text { negociada no mês t. }\end{array}$ \\
\hline Negócios & Quantidade de negócios realizados com ações da empresa em $t$. \\
\hline Volume & Volume financeiro total negociado pelas ações da empresa em $t$. \\
\hline Títulos & Quantidade de títulos negociados por uma determinada ação em $t$. \\
\hline Turnover & $\begin{array}{l}\text { Razão entre a quantidade de ações negociadas e a quantidade de ações em } \\
\text { circulação. }\end{array}$ \\
\hline
\end{tabular}

Fonte: os autores.

Por questões de praticidade, optou-se por apresentar neste estudo um exemplo da construção fatorial considerando a liquidez do mercado brasileiro como um todo. Desse modo, a liquidez do mercado para um dado período $t$ foi obtida pela média de todas as ações a cada período, para cada uma das cinco proxies utilizadas, obtendo-se, assim, cinco séries temporais de 199 observações que vão de dezembro de 1994 a junho de 2011, perfazendo mais de 16 anos de negociações no mercado acionário brasileiro.

\subsection{CONSTRUÇÃO FATORIAL}

A partir disso, o fator foi calculado por meio do procedimento de análise fatorial de séries temporais (TSFA), como introduzido por Gilbert e Meijer (2005). A TSFA fornece um método para estimar variáveis econômicas latentes não observadas, os fatores, sendo, desse modo, uma extensão da análise fatorial tradicional ao trabalhar com séries de tempo em vez de dados de corte.

O nome análise fatorial de séries temporais é usado para distinguir essa técnica da análise fatorial dinâmica (DFA), a qual possui a desvantagem de que um modelo independente deve ser especificado para os fatores. Consequentemente, os parâmetros estimados e os fatores resultantes dependem da modelagem dinâmica utilizada. Com tal procedimento se tornando frequentemente indesejável, uma vez que as diferenças entre os modelos econômicos podem ser exageradas ou obscuras em razão dos diferentes resultados obtidos pelos fatores. Assim, a TSFA trata a construção do fator simplesmente como um problema de mensuração. Desse modo, o modelo dinâmico dos fatores não é assumido, o que permite a possibilidade de que diversos modelos econômicos possam utilizar a mesma construção fatorial (GILBERT; MEIJER, 2005, 2006). 
A análise fatorial tradicional não trabalha diretamente com séries temporais, já que esta assume que suas observações são independentes e identicamente distribuídas. Nesse sentido, séries econômico-financeiras, as quais, em geral, são serialmente dependentes, não satisfazem as premissas básicas. Um modelo genérico de análise fatorial para séries temporais, conforme especificado por Gilbert e Meijer (2005, 2006) é apresentado a seguir.

$$
\mathrm{yt}=\alpha+\mathrm{B} \xi \mathrm{t}+\varepsilon \mathrm{t}
$$

Na Equação 1, os $k$ processos de interesse não observados (os fatores) para uma amostra de $T$ períodos são indicados por , $t=1, \ldots, T, i=1, \ldots, k$. Os $M$ processos observados (os indicadores) são denotados por , $t=1, \ldots, T, i=1, \ldots, M$. Os fatores e indicadores para o período $t$ são representados pelos vetores e , respectivamente. Desse modo, assume-se que exista um modelo de mensuração relacionando os indicadores aos fatores, sendo o intercepto do modelo, $B$ a matriz paramétrica $M \times k$ das cargas fatoriais e o vetor aleatório dos erros de mensuração, o resíduo do modelo.

Séries temporais são geralmente integradas de ordem 1 quando em nível e estacionárias em primeira diferença. Nesse contexto, a fim de evitar problemas de não estacionariedade, além de padronizar a análise, foi trabalhado com as primeiras diferenças dos logaritmos das medidas utilizadas, gerando, assim, séries de retornos das variáveis, o que, segundo Tsay (2010), é a prática padrão para obtenção de séries financeiras estacionárias. Essa transformação ocorreu conforme a Equação 2.

$$
\ln \Delta X_{t}=\ln X_{t}-\ln X_{t-1}
$$

Na Equação 2, é o logaritmo natural da diferença da variável em $t$ e $t$-1. Conforme Gilbert e Meijer (2005), o modelo fatorial genérico apresentado em (1) pode ser obtido tanto com variáveis diferenciadas quanto com as séries originais, mantendo o mesmo parâmetro $B$. Desse modo, o modelo com as variáveis em primeira diferença é obtido com a mesma matriz de cargas fatoriais. Gilbert e Meijer (2005) apresentam esse procedimento como em (3) e (4).

$$
y_{t}-y_{t-1}=\left(\alpha-\alpha_{t-1}\right)+B\left(\xi_{t}-\xi_{t-1}\right)+\left(\varepsilon_{t}-\varepsilon_{t-1}\right)
$$

ou 


$$
D y_{t}=\tau_{t}+B D \xi_{t}+D \varepsilon_{t}
$$

Na Equação 4, é a diferença do indicador em $t$ e $t-1$, é a diferença do fator em $t$ e $t$-1, é um termo constante, é um parâmetro idêntico ao da Equação 1, que indica as cargas fatoriais, e é o resíduo do modelo. No que segue, são apresentados os pressupostos assumidos pelo modelo para obtenção de estimadores consistentes:

$\Sigma_{t}^{\mathrm{T}}={ }_{1} D \xi / T \stackrel{p}{\rightarrow} k$, a média do fator existe e é finita;

$\Sigma_{t}^{\mathrm{T}}={ }_{1} D \varepsilon / T \rightarrow=0$, a esperança do resíduo é igual a zero;

$\left.\Sigma_{t}^{\mathrm{T}}={ }_{1} D \xi_{t}-k\right)\left(D \xi_{t}-k\right)^{\prime} / T \stackrel{p}{\rightarrow} \Phi$, a covariância do fator existe, é finita e definida positiva; $\Sigma_{t}^{T}={ }_{1} D \varepsilon_{t} D \varepsilon^{\prime} / T \stackrel{p}{\rightarrow} \Omega$, a covariância do resíduo existe, é finita e definida positiva; $\Sigma_{t}^{\mathrm{T}}={ }_{1}\left(D \xi_{t}-k\right) D \varepsilon^{\prime} / T$, a covariância do resíduo com o fator é zero.

Não há suposições explícitas sobre a autocorrelação dos dados diferenciados, o que permite a existência de dependência serial nas variáveis. Já a presença de raiz unitária viola os pressupostos, porém as séries podem ser diferenciadas até a sua eliminação para que os pressupostos voltem a ser aplicados. As médias e variâncias, por sua vez, são delimitadas apenas para se obter um limite de probabilidade, não havendo a necessidade de serem constantes ao longo do tempo.

A média amostral e a covariância das séries diferenciadas são denotadas por e $S_{D y}$, respectivamente. Isto é:

$$
\begin{aligned}
& \overline{D y} \equiv \frac{1}{T} \sum_{T=1}^{T} D y_{t} \\
& S_{D y} \equiv \frac{T}{1} \sum_{i=1}^{T}\left(D y_{t}-D_{y}\right)\left(D y_{t}-\overline{D_{y}}\right)^{\prime}
\end{aligned}
$$

A partir dos pressupostos anteriores, segue-se que

$$
\overline{D_{y}} \stackrel{p}{\rightarrow} \mu \equiv \tau+B_{k}
$$

e

$$
S_{D y} \stackrel{p}{\rightarrow} \Sigma \equiv B \Phi B^{\prime}+\Omega
$$


Dados os estimadores $\hat{B}, \hat{\Phi}$ e $\hat{\Omega}$, os estimadores para $\tau$ e/ou $k$ podem ser obtidos de (5). O número de médias amostrais nessa equação é menor que o número de parâmetros, e, portanto, algumas limitações devem ser impostas. Em uma análise fatorial tradicional, os interceptos são parâmetros livres, de modo que as médias dos fatores podem ser arbitrariamente restringidas a zero, com a restrição $k=0$ e o estimador $\hat{\tau}=\overline{D y}$. Quando $\tau$ e $k$ é diferente de zero, um estimador consistente para $k$ é o estimador de mínimos quadrados generalizados:

$$
\hat{k}=\left(\hat{B}^{\prime} \hat{\Omega}^{-1} \hat{B}\right)^{-1} \hat{B}^{\prime} \hat{\Omega}^{-1} \overline{D y}
$$

No caso específico, é utilizado o estimador de máxima verossimilhança encontrado pela minimização de (7):

$$
L \equiv \log \operatorname{det} \Sigma+\operatorname{tr}\left(\Sigma^{-1} S_{D y}\right)
$$

Na Equação 7, é uma função dos parâmetros, como em (6), com os estimadores consistentes não sendo totalmente de máxima verossimilhança, mas de quase máxima verossimilhança no sentido de White (1982). Mais informações sobre a construção do modelo e sobre o procedimento de estimação podem ser vistos em Gilbert e Meijer $(2005,2006)$. Ademais, todas as estimações descritas anteriormente são realizadas por meio do software livre R com o auxílio do pacote Time Series Factor Analysis (TSFA), criado e apresentado por Gilbert e Meijer (2006).

\section{ANÁLISES DOS RESULTADOS}

Inicialmente, com o intuito de evidenciar a evolução das séries estudadas, foram apresentados gráficos das variáveis em nível e em primeira diferença de logaritmos (log-retornos). A Figura 1 expõe o comportamento temporal dessas variáveis. 
Figura 1 - Evolução temporal da média do número de negócios, do volume negociado, do número de títulos, do turnover e do bid-ask spread e seus respectivos log-retornos para o período de janeiro de 1995 a junho de 2011
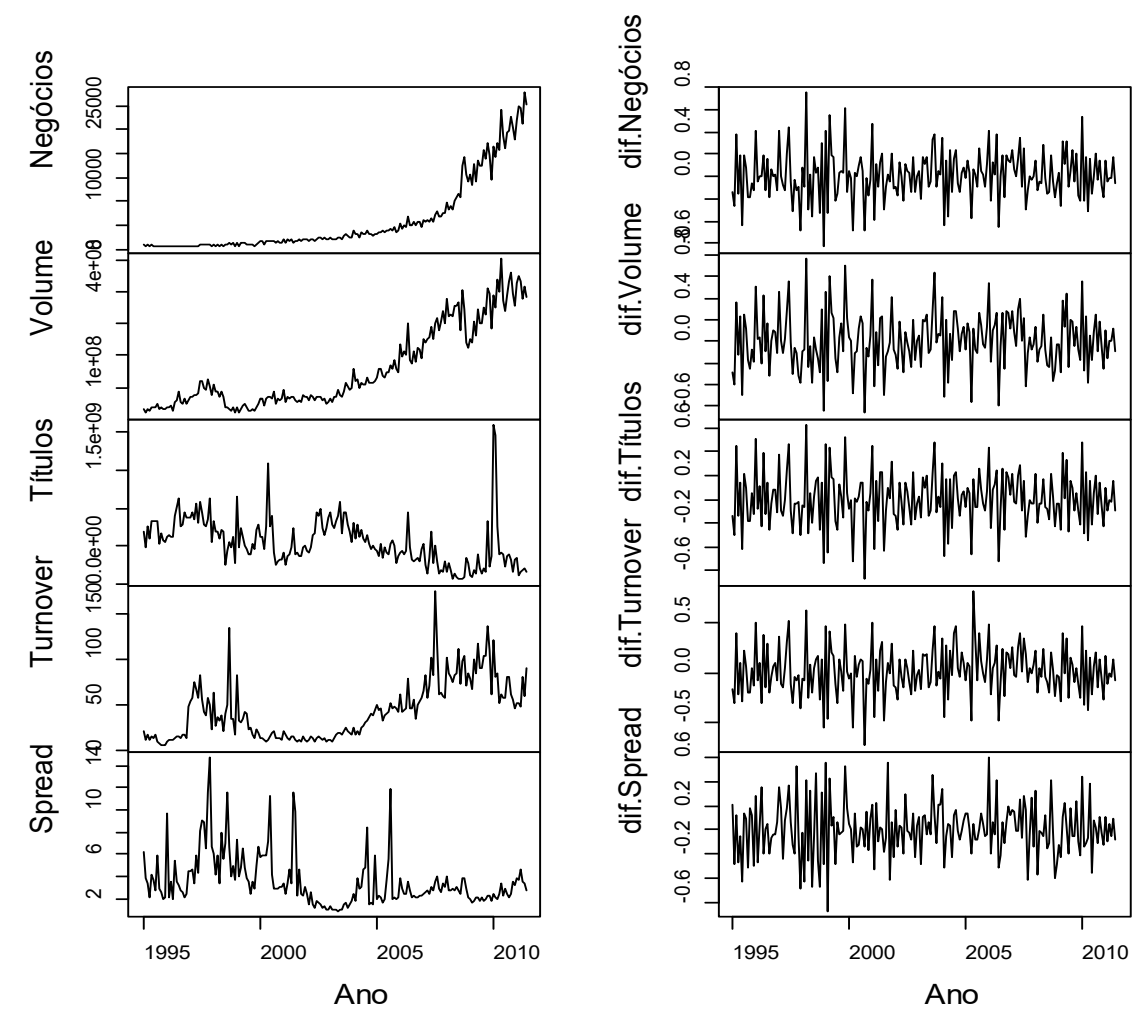

Fonte: os autores.

Ao se observar o comportamento das séries, percebe-se que o Bid-Ask Spread apresentou certa volatilidade ao longo do período, porém se estabilizou em meados de 2005 e apresentou valores reduzidos até o fim da amostra. Esse comportamento demonstra maior consolidação do mercado brasileiro a partir desse período, uma vez que a diferença média do preço de compra e venda das ações se reduziu.

Quanto ao número de negócios e ao volume, estes exibiram uma tendência crescente a partir de 2005, o que reitera a maior consolidação do mercado acionário brasileiro a partir desse período. A quantidade de títulos negociados, por sua vez, permaneceu estável ao longo do período, o que, em relação ao aumento no número de negócios e ao volume negociado, indica uma elevação nos preços das ações e no número de vezes em que são negociadas. O turnover (multiplicado por 100 para facilitar 
a exposição gráfica) expõe a relação entre a quantidade de ações negociadas em determinado mês e o número de ações em circulação no período e permitiu demonstrar que a intensidade com que as ações são negociadas também se elevou após 2005, embora de modo menos intenso.

Na segunda parte da Figura 1 é exposta a evolução temporal das variáveis em log-retorno, como utilizadas na estimação do modelo. De modo geral, todas possuem um comportamento típico de séries estacionárias, o que é esperado, uma vez que a transformação em log-retorno é o procedimento padrão empregado na literatura para obtenção de séries financeiras estacionárias, o que permite, além de evitar problemas de inferência estatística, padronizar os resultados obtidos.

Dando continuidade à análise, são apresentadas na Tabela 1 as estatísticas descritivas das séries em nível da amostra utilizada. Como pode ser observado, as medidas de liquidez utilizadas apresentaram médias e desvios padrão de diferentes magnitudes, uma vez que captam diferentes características do mercado e não possuem um comportamento padronizado como o das variáveis em log-retorno. A maior diferença entre preços de compra e venda foi da Companhia Paulista de Força e Luz, em agosto de 2005. A Petrobras, por sua vez, obteve o maior volume negociado e número de negócios realizados durante o período. Além dessas características individuais de cada medida, as variáveis apresentaram um comportamento positivamente assimétrico, havendo maior frequência de observações à esquerda da média. Ademais, as medidas demonstraram uma curtose leptocúrtica com a frequência mais concentrada, o que, de modo geral, indica que nenhuma variável apresenta um comportamento probabilístico característico de séries normalmente distribuídas.

Tabela 1 - Estatísticas descritivas das variáveis em nível

\begin{tabular}{lrrrrrr}
\hline Variável & \multicolumn{1}{c}{ Média } & Desvio padrão & \multicolumn{1}{c}{ Mínimo } & \multicolumn{1}{c}{ Máximo } & Assimetria & Curtose \\
\hline Spread & 3,3941 & 24,7111 & 0,0000 & 1803,24 & 31,4341 & 1441,46 \\
Negócios & 7994,92 & 27734,6 & 1,0000 & 681805 & 8,8927 & 113,473 \\
Volume & 192400000 & 867008000 & 134,0000 & 22828100000 & 13,2765 & 230,596 \\
Titulos & 505856000 & 5381490000 & 2,0000 & 345615000000 & 26,6950 & 1178,76 \\
Turnover & 0,0511 & 0,2908 & $<0,0001$ & 23,1146 & 32,8661 & 1694,92 \\
\hline Fonte: os autores. & & & & &
\end{tabular}

Prosseguindo com essa análise descritiva, é apresentada na Tabela 2 a matriz de correlação das variáveis. Pestana e Gageiro (2000) afirmam que, por convenção, um coeficiente de correlação menor que 0,2 indica uma associação muito baixa, entre 
0,2 e 0,3, baixa, entre 0,4 e 0,69, moderada, entre 0,7 e 0,89, alta e, por fim, entre 0,9 e 1 , uma associação muito alta.

Nesse sentido, os resultados indicam uma elevada associação entre todas as medidas de liquidez escolhidas para a construção fatorial. A correlação entre as variáveis variou positivamente de 61 a 95\%. A menor associação encontrada foi de $61 \%$ entre o spread e o volume negociado, já a maior ocorreu entre o volume e o número de títulos. De modo geral, os resultados da matriz de correlação se mostram propícios a uma construção fatorial, já que todas as variáveis analisadas apresentam um forte relacionamento entre si.

Tabela 2 - Matriz de correlação das variáveis em log-retorno empregadas nas estimações

\begin{tabular}{lcrrrr}
\hline & Spread & \multicolumn{1}{l}{ Negócios } & \multicolumn{1}{l}{ Volume } & \multicolumn{1}{l}{ Títulos } & \multicolumn{1}{c}{ Turnover } \\
\hline Spread & 1,0000 & 0,6839 & 0,6190 & 0,6519 & 0,6215 \\
Negócios & & 1,0000 & 0,9425 & 0,9449 & 0,8931 \\
Volume & & & 1,0000 & 0,9506 & 0,8978 \\
Títulos & & & & 1,0000 & 0,9444 \\
Turnover & & & & & 1,0000 \\
\hline Fon
\end{tabular}

Fonte: os autores.

Na sequência é apresentado o gráfico dos autovalores obtidos para a seleção do número de fatores a ser escolhido. Os autovalores da matriz de correlação dos dados dão uma ideia aproximada do número de fatores a considerar. Esses valores são: 4,29; 0,50; 0,12; 0,05; e 0,03, expostos no Gráfico 1. Como regra convencional, tem-se que o número de fatores deve ser igual ao número de valores maiores que 1,0. Desse modo, foi usado o primeiro fator como representativo da liquidez acionária.

Gráfico 1 - Scree Plot dos autovalores estimados

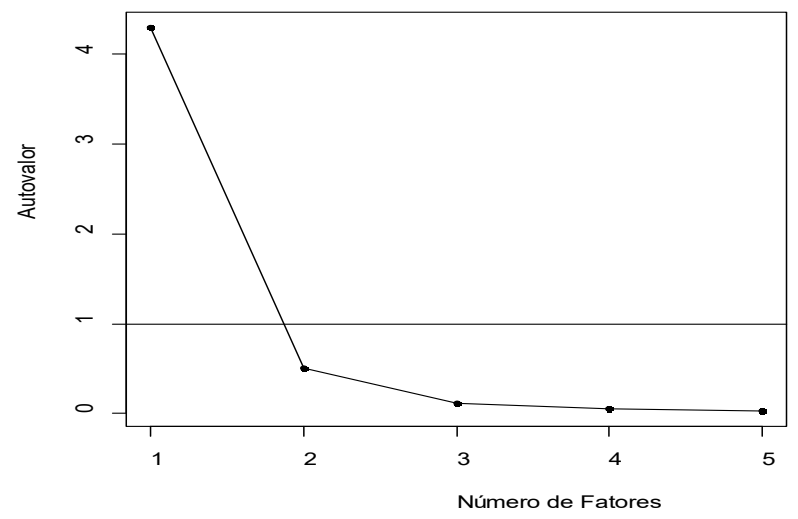

Fonte: os autores. 
No que segue, apresenta-se a Tabela 3 com as cargas fatoriais padronizadas, as comunalidades obtidas e a correlação entre o fator gerado e as medidas de liquidez. As cargas fatoriais padronizadas (dimensionadas para que a variância das séries seja 1,0) são tradicionalmente mais fáceis de interpretar, já que uma carga maior (em valores absolutos) indica uma relação mais intensa. Na coluna do meio são apresentadas as comunalidades. A comunalidade de um indicador é sua variância comum (ou seja, a variância explicada pelos fatores) expressa como uma fração de sua variância total. Assim, é o coeficiente de determinação da regressão contra os fatores.

Tabela 3 - Cargas fatoriais padronizadas, comunalidades e correlação entre o fator e as medidas de liquidez

\begin{tabular}{lrrr}
\hline \multicolumn{1}{c}{ Variável } & Cargas fatoriais padronizadas & Comunalidades & $\begin{array}{c}\text { Correlação } \\
\text { (Fator) }\end{array}$ \\
\hline Fator & - & - & 1,0000 \\
Negócios & 0,9534 & 0,9091 & 0,9530 \\
Volume & 0,9780 & 0,9564 & 0,9606 \\
Títulos & 0,9975 & 0,9950 & 0,9988 \\
Turnover & 0,9761 & 0,9529 & 0,9524 \\
Spread & 0,6953 & 0,4834 & 0,6575 \\
\hline Fonte: os autores
\end{tabular}

A regra habitual é que uma carga fatorial padronizada inferior a 0,3 não é suficientemente grande para ser considerada importante. Já quanto às comunalidades, estas podem se mostrar menores do que as tipicamente encontradas nas análises fatoriais tracionais. Contudo, conforme explicam Gilbert e Meijer (2005), isso se deve ao tipo de dados utilizados, isto é, séries temporais diferenciadas.

Observando a Tabela 3, as cargas fatoriais padronizadas variaram entre 0,69 e 0,99. O número de negócios, volume, número de títulos e turnover obtiveram uma participação muito similar na formação do fator, com todos os valores sendo superiores a 0,95, dado um valor máximo de 1,0 para as cargas padronizadas. O spread, por sua vez, apresentou a menor participação na formação do fator, a qual foi de 0,69. No que diz respeito às comunalidades, estas representam a variância comum entre os fatores e cada uma das variáveis utilizadas em sua formação. Os valores obtidos foram de 0,48 a 0,99. Novamente, o número de títulos se destacou, sendo a variável com maior variância comum com o fator e o spread com menor relacionamento.

Ainda, tem-se a matriz de correlação entre o fator estimado e as proxies utilizadas na sua formação, a qual visa ilustrar a capacidade do fator em substituir essas medidas em possíveis modelos econométricos. A correlação variou entre 0,65 e 0,99. Da mesma forma que para as comunalidades, o número de títulos apresentou a maior 
correlação com o fator, e o spread, o menor relacionamento. Os valores elevados da correlação entre o fator e as medidas individuais destaca a capacidade, em termos de variância, de o fator gerado representar as medidas individuais. Além disso, para ilustrar esse relacionamento é exposta a Figura 3, contendo o fator e a evolução deste, individualmente, com cada medida de liquidez.

Figura 2 - Evolução temporal do fator estimado e do relacionamento do fator com as medidas de liquidez
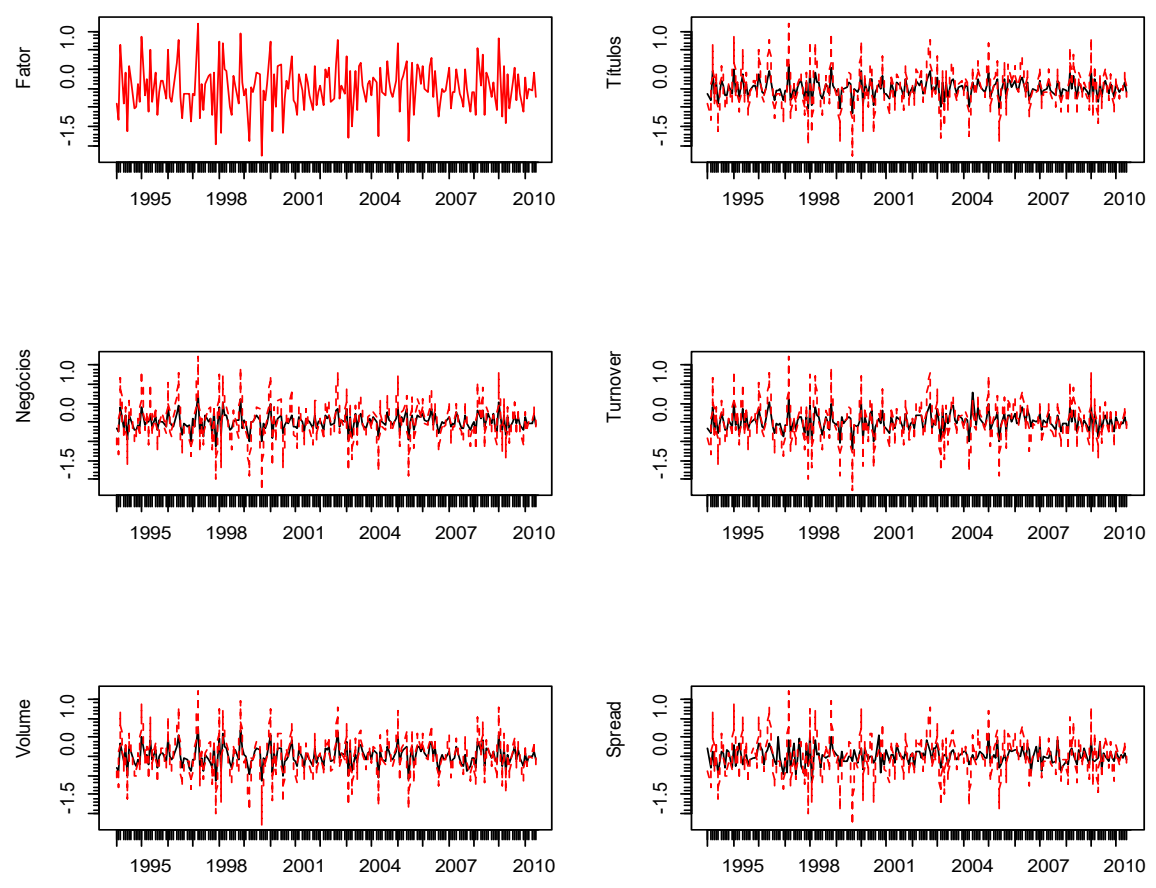

Fonte: os autores.

A respeito da Figura 2, esta apresenta o fator no primeiro gráfico. Os demais, por sua vez, demonstram a evolução de cada medida de liquidez (em preto) com o fator (em vermelho pontilhado). De modo geral, o fator gerado evolui de forma similar com as demais medidas. Além disso, apresenta um comportamento típico de séries estacionárias, o que permite seu emprego em modelos econométricos que exigem esse pressuposto. 


\section{CONSIDERAÇÕES FINAIS}

Este estudo teve o objetivo de sugerir o emprego de uma metodologia alternativa para mensuração da liquidez em mercados acionários. Para tanto, o procedimento empregado foi a Análise Fatorial de Séries Temporais (TSFA), proposta por Gilbert e Meijer (2005). Com base nesse modelo, foram utilizados dados da negociação de ações da BM\&FBOVESPA e cinco diferentes proxies de liquidez para a construção fatorial.

Como constatações, o estudo permitiu observar a possibilidade de se combinarem diferentes medidas de liquidez para a formação de uma única variável. Essa variável (o fator) demonstrou possuir uma forte associação com as proxies utilizadas em sua construção, o que torna plausível a sua aplicação em modelos econométricos que estudem liquidez. Como vantagens, esse procedimento permite eliminar problemas de colinearidade em estimações estatísticas e possibilita que análises de investimento utilizem uma única variável para mensurar a liquidez ao invés de várias simultaneamente. Além disso, o fator gerado possui um comportamento típico de séries estacionárias, o que permite seu emprego em análises de investimento que utilizam séries temporais, já que este é um pressuposto comum para esse tipo de modelagem. Outro ponto de destaque é a capacidade de a construção fatorial ser replicada para novos dados, já que não é assumido um modelo dinâmico para os fatores, o qual é tratado simplesmente como um problema de mensuração.

Por fim, apresentam-se as seguintes sugestões para estudos futuros: replicar o procedimento para dados de diferentes frequências e mercados, a fim de verificar a viabilidade da construção fatorial e empregar o fator construído e as proxies utilizadas em modelos estatísticos que estudem a liquidez, para que possam ser comparadas as diferenças e semelhanças entre eles.

\section{REFERÊNCIAS}

AMIHUD, Y. Iliquidity and stock returns: cross-section and time series effects.

Journal of Financial Markets, v. 5, i. 1, p. 31-56, 2002.

AMIHUD, Y.; MENDELSON, H. Asset pricing and the bid-ask spread. Journal of Financial Economics, v. 17, i. 2, p. 223-249, 1986.

AMIHUD, Y.; MENDELSON, H. Liquidity, assets prices and financial policy. Financial Analysts Journal, v. 47, i. 6, p. 56-66, 1991. 
BLACK, F. Towards a fully automated exchange. Financial Analysts Journal, v. 27, p. 29-35, 1971.

BRUNI, A. L.; FAMÁ, R. Liquidez e avaliação de ativos financeiros: evidências empíricas na Bovespa (1988-1996). In: ENCONTRO ANUAL DA ASSOCIAÇÃO NACIONAL DO PROGRAMA DE PÓS-GRADUAÇÃO EM ADMINISTRAÇÃO, 22., 1998, Foz do Iguaçu. Anais... Foz do Iguaçu, 1998.

CHORDIA, T.; ROLL, R.; SUBRAHMANYAN, A. Commonality in liquidity. Journal of Financial Economics, v. 56, i. 1, p. 3-28, 2000.

CORREIA, L. F.; AMARAL, H. F. A influência da liquidez das ações sobre o retorno no mercado acionário brasileiro. In: ENCONTRO BRASILEIRO DE FINANÇAS, 12., 2012, São Paulo. Anais... São Paulo, 2012. Disponível em: <http://bibliotecadigital.fgv.br/ocs/index.php/ebf/12EBF/schedConf/presentations >. Acesso em: 15 nov. 2015.

CORREIA, L. F.; AMARAL, H. F.; BRESSAN, A. A. O efeito da liquidez sobre a rentabilidade de mercado das ações negociadas no mercado acionário brasileiro. Revista de Administração e Contabilidade da Unisinos - BASE, v. 5, n. 2, p. 111118, 2008.

DATAR, V. T.; NAIK, N. Y.; RADCLIFFE, R. Liquidity and stock returns: an alternative test. Journal of Financial Markets, v. 1, p. 203-219, 1998.

FAMA, E. F.; FRENCH, K. R. Common risk factors in the returns on stocks and bonds. Journal of Financial Economics, v. 33, i. 1, p. 3-56, 1993.

FAMA, E. F.; MACBETH, J. Risk, return and equilibrium: empirical tests. Journal of Political Economy, v. 71, p. 607-636, 1973.

GILBERT, P. D.; MEIJER, E. Money and Credit Factors. Bank of Canada Working Paper 2006-3, 2006. Disponível em: <http://www.bankofcanada.ca/wp-content/ uploads/2010/02/wp06-3.pdf>. Acesso em: 10 out. 2014

GILBERT, P. D.; MEIJER, E. Time Series Factor Analysis with an Application to Measuring Money. Research Report 05F10. University of Groningen, Nov. 2005. Disponível em: <http://som.eldoc.ub.rug.nl/reports/themeF/2005/05F10/>. Acesso em: 10 out. 2014. 
HOUAISS, A.; VILLAR, M. S. Dicionário Houaiss da língua portuguesa. Rio de Janeiro: Objetiva, 2009.

KYLE, A. S. Continuous Auctions and Insider Trading. Econometrica, v. 53, p. 1315-1335, 1985.

LIU, W. A liquidity-augmented capital asset pricing model. Journal of Financial Economics, v. 82, p. 631-671, 2006.

MACHADO, M. A. V.; MACHADO, M. R. Liquidez e precificação de ativos: evidências do mercado brasileiro. Brazilian Business Review, v. 11, n. 1, p. 73-95, 2014.

MACHADO, M. A. V.; MEDEIROS, O. R. de. Modelos de precificação de ativos e o efeito liquidez: evidências empíricas no mercado acionário brasileiro. Revista Brasileira de Finanças, v. 9, n. 3, p. 383-412, 2011.

MACHADO, M. A. V.; MEDEIROS, O. R. Existe o efeito liquidez no mercado acionário brasileiro? Brazilian Business Review, v. 9, n. 4, p. 28-51, 2012.

MINARDI, A. M.; SANVICENTE, A. Z. MONTEIRO, R. Spread de compra e venda no mercado acionário brasileiro, liquidez, assimetria de informação e prêmio por liquidez. In: ENCONTRO BRASILEIRO DE FINANÇAS DA SOCIEDADE BRASILEIRA DE FINANÇAS, 5., 2005, Rio de Janeiro. Anais... Rio de Janeiro, 2005.

PASTOR, L.; STAMBAUGH, R. F. Liquidity risk and expected stock returns. Journal of Political Economy, v. 111, p. 642-685, 2003.

PESTANA, M. H.; GAGEIRO, J. N. Análise de Dados para Ciências Sociais: A complementaridade do SPSS. 2. ed. Lisboa: Edições Sílabo, 2000.

RIGHI, M. B.; VIEIRA, K. M. Verifying the presence of the liquidity premium in the Brazilian market through different time scales. African Journal of Business Management, v. 1, 2012.

SADKA, R. Liquidity risk and accounting information. Journal of Accounting and Economics, v. 52, i. 2-3, p. 144-152, 2011.

SARR, A.; LYBEK, T. Measuring liquidity in financial markets. International Monetary Fund. Working Paper. 2002. Disponível em: <http://www.imf.org/external/pubs/ft/wp/2002/wp02232.pdf>. Acesso em: 15 nov. 2015 
TSAY, R. S. Analysis of financial time series. 3. ed. John Wiley \& Sons, 2010.

VICTOR, F.; PERLIN, M. S.; MASTELLA, M. Comunalidades na Liquidez - Evidências e Comportamento Intradiário para o Mercado Brasileiro. Revista Brasileira de Finanças, v. 11, n. 3, p. 375-398, 2013.

VIEIRA, K. M.; JUSTEN JÚNIOR, A. A.; RIGHI, M. B. O papel da liquidez e suas múltiplas dimensões no retorno das ações: um estudo com dados em painel do mercado brasileiro. Contextus - Revista Contemporânea de Economia e Gestão, v. 13, n. 2, p. 7-35, 2015.

VIEIRA, K. M.; MILACH, F. T. Liquidez/Iliquidez no mercado brasileiro: comportamento no período 1995-2005 e suas relações com o retorno. Revista de Administração e Contabilidade da Unisinos - BASE, v. 5, n. 1, p. 5-16, 2008.

WHITE, H. Maximum likelihood estimation of misspecified models. Econometrica, v. 50, p. 1-25, 1982.

\section{Como citar este artigo:}

\section{ABNT}

SILVEIRA, Vinicius Girardi da et al. Análise fatorial de séries temporais para medidas de liquidez no mercado brasileiro. RACE, Revista de Administração, Contabilidade e Economia, Joaçaba: Ed. Unoesc, v. 16, n. 3, p. 1109-1132, set./dez. 2017. Disponível em: <http://editora.unoesc.edu.br/index.php/race> . Acesso em: dia/mês/ ano.

APA

Silveira, V. G. da, Vieira, K. M., Bender Filho, R., \& Coronel, D. A. (2017). Análise fatorial de séries temporais para medidas de liquidez no mercado brasileiro. RACE, Revista de Administração, Contabilidade e Economia, 16(3), 1109-1132. Recuperado em dia/mês/ano, de http://editora.unoesc.edu.br/index.php/race 